\title{
Identification of Small Unmanned Flying Objects
}

\author{
Lucjan Setlak, Rafał Kowalik
}

\begin{abstract}
Detecting obstacles during the flight and avoiding them is desirable in the case of unmanned aerial vehicles intended for observation of a residential area, refers especially to lightweight micro-aviation vehicles of multi-rotor type, and is also a serious problem because their load capacity is limited, therefore only electronic sensors can be connected to the object. Usually the sensors built into the system are either based on a type vision (monocular or stereo camera) or on a laser camera. However, each of the sensors has its advantages and disadvantages, which is why the article presents the concept of a system for collecting data characterizing the flight of a UAV object and including them in the object identification process. The main purpose of this work is to perform selected studies (analysis, mathematical model, simulations) in the field of identification of small unmanned flying objects. A dynamic model describing UAV motion was developed, which took into account flight parameters using various identification methods. The structure of this work is contained in four chapters, in which, among others, the second chapter deals with the review of existing identification systems for small UAV objects, based on an analysis of the literature on the subject of research. The third chapter covers the issues related to aerodynamics and mechanics of small UAV objects and concerns linear longitudinal equations of UAVs based on Newton's second law. This chapter also describes the algorithm used for dynamic description along with incorrect filtering of "on-line" learning patterns and characterizes the least squares recursive method used for the simulation. Based on the analysis, mathematical models created, simulations performed and the results obtained based on them, practical conclusions presented in the final part of the article were formulated.
\end{abstract}

Keywords - identification, unmanned aerial vehicle, small flying objects, simulations

\section{INTRODUCTION}

$\mathrm{N}_{\mathrm{p}}^{\mathrm{c}}$ OWADAYS small unmanned drones are newly created personal aircraft used for civilian applications and research. These objects can provide scientists with multiple images with a shorter time of setting and returning data, which is crucial for dynamic, time-sensitive data analysis. UAV (Unmanned Aerial Vehicles) objects are also perfectly adapted to research and optimization in agriculture, characterized by small areas of data collection, high resolution and fast data availability.

They are able to find a gap in resolution for applications in grazing areas, providing the necessary data for the management

Lucjan Setlak is with the Polish Air Force University, Aviation Division, Department of Avionics and Control System, Deblin, Poland (corresponding author to provide phone: +48-261-518-865, e-mail: l.setlak@law.mil.pl).

Rafał Kowalik is with the Polish Air Force University, Aviation Division, Department of Avionics and Control System, Deblin, Poland (phone: +48261-518-824, e-mail: r.kowalik@law.mil.pl). of areas. They are also used to map and detect changes on the coasts and wetlands.

Thanks to their properties, they can provide multispectral images in high resolutions, with the operator retaining full control over the time and place of taking the picture.

However, it should be noted that there are many obstacles that must be overcome so that UAV objects can successfully integrate into the civil airspace. The biggest obstacles and difficulties concern safety, i.e. bypassing obstacles, communication between both manned and unmanned aircraft as well as the reliability of the system and its resistance to various types of failures and any damage [1], [2], [3].

The systems described above are an integral part of effective and safe personal remote sensing. The development of such systems requires well-designed and characterized dynamic models [4], [5]. The dynamic model for traditional aircraft and rotorcraft was analytically determined by the second law of Newton's dynamics. The parameters of this dynamic model are usually determined by expensive and time-consuming tests in the wind tunnel.

These methods, despite being very useful and have many advantages, have limitations when used for small and very small aircraft due to several key differences, which include:

- low Reynolds number and speed,

- increased dynamic speed due to smaller moments of inertia,

- domination of forces and moments of driving dynamics in comparison with forces and aerodynamic moments,

- $\quad$ asymmetrical and untypical shapes,

- cases in which it is not possible to characterize all flight regimes, i.e. the transition from pre-flight mode to fixed flight, aerobatic maneuvers and reverse helicopter flight.

As an alternative to the analytical methods and testing in the wind tunnel, system identification provides several well-suited methods for developing dynamic models of the system and identifying their parameters. There are several methods of system identification that meet the requirements for their application to small UAV objects.

Through the system identifier, the dynamic flight model can be determined on the basis of the flight data, and can then be used to develop and check the automatic pilot control systems. The identification system is an indispensable tool for modeling, simulating and developing controllers of small UAV objects [6], [7]. 
The main purpose of the work is to develop and implement the method of system identification using the smallest recursive squares with the wrong "on-line" learning program. This method is used on small drones with a fixed wing, using one of the cheapest sensors.

\section{REVIEW OF EXISTING IDENTIFICATION SYSTEMS FOR SMALL DRONES}

\section{A. Identification System}

System identification is the process of determining the mathematical model of the dynamic system by analyzing the measured input signals and output states of the system. For this purpose, estimation was used. Instead of estimating the system state or location of controllers, the system uses inputs and positions to develop a model that describes the relationship between the input signals and the system response.

In the case of the specificity of UAV objects, the process of system identification begins with the design of input signals. Then the pilot or the automatic pilot performs maneuvers to stimulate dynamicly the UAV object. Signals given to actuators and control surfaces are recorded.

The actual deviation of the control surfaces can also be recorded. Then, various types of sensors record the current state of the UAV object: acceleration, velocity, angular velocities, positions of aerodynamic angles and angles relative to the surface of the Earth.

The dynamic model is recognized from the above data. If the structure of the model is known, then its parameters can be identified. This is referred to as parameter estimation, which is a more specific type of system identifier. The response of the model is checked using test data. Authentication uses a set of input data separated from the test data to generate the model. If the test data and the identified model match, it is possible to use it.

However, if the model does not adequately predict flight characteristics, then the identification method and input signals must be re-examined and tested. This process is described below.

1. Input signals.

2. Database.

3. Selection of the model structure.

4. Choosing the system identification method.

5. Optimization of the model using the method of system identification, model structure and test data.

The above-mentioned elements of the system identifier are characterized in more detail in the subsequent chapters of the work. These chapters are designed to provide the basics of knowledge about the actual implementation of the system identifier, specifically focused on the dynamics of the UAV object [8], [9].

\section{B. Input signals}

The input signals are probably the most important part of the system identifier, because they affect all other aspects of the identification process. These signals are used to excite dynamic system modes.
By analyzing the system inputs and responses, the model and model parameters can be determined. If the dynamic modes of the system are not excited, the dynamic modes will not be displayed in the test data and will not be able to be identified in the model.

For this reason, it is very important to have inputs specially designed to stimulate the dynamics of the system. However, simple dynamic stimulation does not guarantee that the identification method will determine the dynamic model.

Dynamic modes must be excited or persistently excited so that all the necessary modes are involved. In order for the identification method to have time to identify the model, it may be necessary to permanently excite the modes. This applies to dynamic systems such as fugoid oscillations for fixed wings.

In contrast, inputs can be designed if there is sufficient information about system dynamics. This is usually not the case when identifying a model for a UAV object, especially when creating a prototype. For general inputs, it is recommended to perform a frequency review for the identification system and doublets in order to verify the model.

Frequency deviations may be constant or varying depending on the dynamics that must be stimulated. The frequency starts at a given low frequency and increases up to the desired stop frequency (Fig. 1).

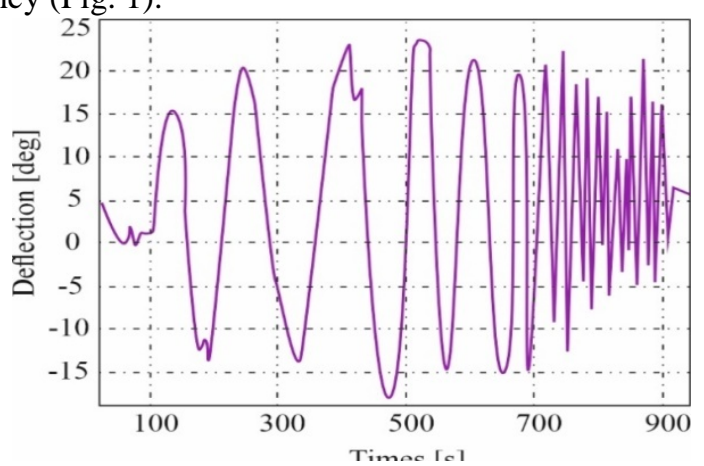

Fig. 1. Changing the frequency for the system identifier from the flight data

These signals can be set manually by the pilot or automatically by the automatic pilot. The input signals for the identification method are usually given by the pilot to keep the drone under full control (Fig. 2). Work is underway to create an optimal automatic pilot maneuver to determine the identification method [10], [11], [12].

In summary, the following points should be considered when developing input data for the system identification structure:

1. Frequency range.

2. Stability of excitation.

3. Excitation time.

4. Types of entries.

5. Model verification methods. 


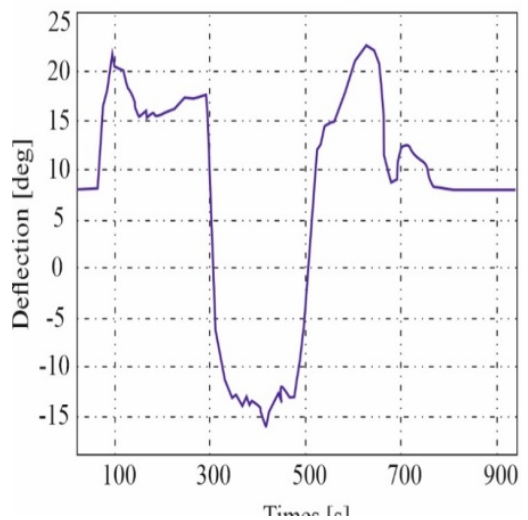

Fig. 2. Doublet for model verification from flight data

\section{Preparation and collection of test data}

There are several issues that must be considered when determining the requirements for the identification method. One of the challenges of the system is to use real flight data, whereby flight data can be very noisy. The amount of noise depends on the type of sensor and its integration with avionics, the drone structure and the type of drive.

For example, an inertial unit of measure registers acceleration as a result of changes in aerodynamic forces and moments and structural vibrations caused by the propulsion system. This type of vibration can be observed by analyzing the following figures (Figs. 3-6). Figures 3 and 5 show acceleration during frequency changes, and noise from the drive system is illustrated in figures 4 and 6 .

It should be noted that when accelerating in the direction of the $Z$ axis, the signal to noise ratio is high and the movement is relatively slow compared to the noise.

In this case, you can use a low-pass filter to eliminate noise from the drive. It is suggested to use the same filter in all input and output signals to prevent signal distortion.

In order to collect test data, the sampling frequency of the sensor should be taken into account. This is due to the fact that if the sensor has a lower sampling rate than the frequency of dynamic vibrations of the system, the identification method may not recognize the model and its parameters.

Sensor type requirements are based on the model, input and output data needed to identify the method. Sensors should be characterized before testing in flight to ensure data reliability. Other sensors or filtering should be considered if the sensor is inherently noisy or a certain part of the data does not have sufficient resolution.

On the basis of the analysis of the literature on the subject of research, the sources of data collection for the UAV object can be grouped in the following way as a method of identification [13], [14], [15]:

1. Inertial measuring unit, system GPS (Global Positioning System) and differential pressure sensor, laser altimeter, ultrasonic altimeter.

2. GPS only.

3. Motion capture systems.

4. Radar.
5. Software in the simulation loop (to compare identification methods).

Typically, the identification system uses data from the inertial measurement unit IMU (Inertial Measurement Unit) and the GPS system, however, it is possible to use other sensors positively.

In summary, the following issues regarding the collection of test data should be taken into account:

1. Noise.

2. Filters.

3. The sampling frequency of the sensor.

4. Types of sensors.

\section{Selection of the model structure}

The choice of the model structure is an important stage in identifying the system. This step requires prior knowledge of system dynamics, understanding of model properties, and understanding of the final application.

Incorrect selection of the model can lead to a situation where only the system dynamics have been described in part. The system identifier may be the most important to choose the model. If aerodynamic coefficients are required, the model must have parameters as part of the structure.

On the other hand, if the model is used to design control systems, an autoregulatory regression model may be appropriate.

\section{E.Choosing the system identification method}

The choice of system identification method depends mainly on the application and system dynamics. System identification methods can be divided into two groups: parametric and nonparametric.

Non-parametric methods identify the system with a pulse or frequency response and do not require prior knowledge of system dynamics or model structure. In turn, the parametric methods assume the structure of the dynamic model, and the model parameters are then identified from the training data.

Parametric models can be divided into the following subgroups:

1. Time-varying and temporally stable systems.

2. Static and dynamic systems.

3. Linear and nonlinear systems.

4. Continuous and discrete systems.

All these groups are interrelated and each of them contains only one particular aspect of the identification method [16], [17]. These categorizations are provided as references for the system identifier methods.

\section{The Results of Simulation Studies AerodynamiC and MechaniCal Models of IDENTIFICATION System}

\section{A. Derivation of 6 Degrees of Freedom of Nonlinear Motion Equat}

The plane motion equations can be divided into a translational motion and rotary motion. The second law of Newton's dynamics can be used to describe both the translational and rotational motion, in the case of this article they were used to develop the motion of a UAV object. 
If the rigid body is installed, the mass of the system can not change over time as in the case of electrically powered drones. Drones with internal combustion drive are subject to the category of the ever-changing mass system and require special care in the case of deriving motion equations.

\section{Coordinate system}

In order to get acquainted with the coordinate system, a set of systems of motion equations is given in the figure below. This coordinate system describes the contractual marking of the acceleration, speed, position and angles used in the motion equation [18], [19].

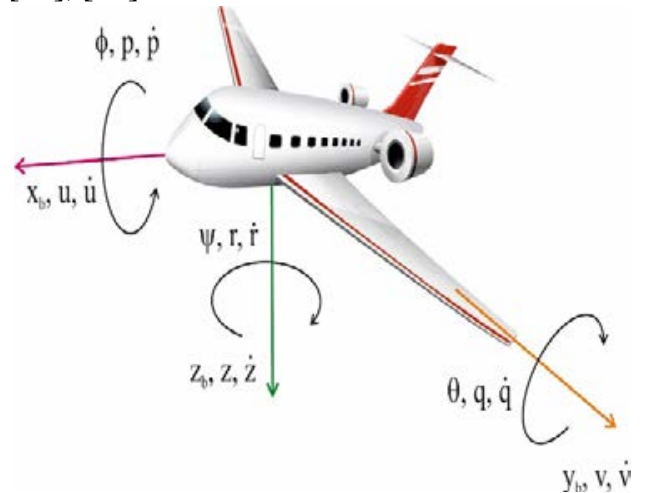

Fig. 3. Coordinate system with accelerations, speeds, positions and angles

\section{B. Derivation of 6 degrees of freedom of non-linear motion equations: translational movement}

Starting from the translational movement in the inertial frame, the rate of change of torque is equal to the sum of forces exerted on the body (1).

$$
\Sigma F_{i}=\frac{d P_{i}}{d t}
$$

where: $P_{i}$ - is the moment of inertia of the translation expressed as (2):

$$
P_{i}=m V_{i}
$$

By substituting equation (2) for equation (1), a formula for translational motion was obtained (3):

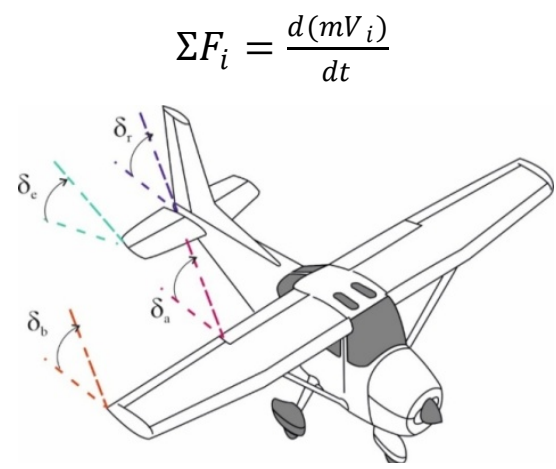

Fig. 4. Deviations of control surfaces

The second law of Newton's dynamics for rigid bodies only applies to solid mass systems. This does not mean that the second law of dynamics can never be applied to a body that loses or gains mass. This means that both the mass and momentum of the entire system must be considered [20], [21].
Because the mass is constant and to avoid confusion with different mass systems, for calculations the mass is taken from a time derivative using the constant factor rule in differentiation (4).

$$
\Sigma F_{i}=m \frac{d V_{i}}{d t}
$$

To simplify the expression and measurement of velocities and angles, the frame velocity vector is expressed in a fixed frame by transformations. This is important because most sensors send data in a frame.

Transformation introduces additional conditions for translational and rotational motion equations. Additional conditions are caused by the body rotating in relation to the inertial frame. The derivative of transformation from the inertial body gives the velocity vector.

Based on the above, the first vector of body speed was determined in the following form (5):

$$
V_{b}=V_{x_{b}} i_{b}+V_{y_{b}} j_{b}+V_{z_{b}} V_{z_{b}} k_{b}
$$

Next, the derivative was determined after the time of velocity vector $V_{b}$ relative to the inertia frame by differentiating the equation (5), which resulted in (6):

$\frac{d V_{b}}{d t_{i}}=\frac{d V_{x_{b}}}{d t_{b}} i_{b}+\frac{d V_{y b}}{d t_{b}} j_{b}+\frac{d V_{z_{b}}}{d t_{b}} k_{b}+V_{x_{b}} \frac{d i_{b}}{d t_{i}}+V_{y_{b}} \frac{d j_{b}}{d t_{i}}+V_{z_{b}} \frac{d k_{b}}{d t_{i}}$

where: $\frac{d}{d t_{i}}$ - is a time derivative relative to the inertial frame.

The first three concepts of the right side of the equation (6) express the change in velocity vector $V_{b}$ in the frame body view.

These three components can be combined at the time of the $V_{b}$ derivative, resulting in (7):

$$
\frac{d V_{b}}{d t_{b}}=\frac{d V_{x_{b}}}{d t_{b}} i_{b}+\frac{d V_{y b}}{d t_{b}} j_{b}+\frac{d V_{z_{b}}}{d t_{b}} k_{b}
$$

The last three components of the right side of the equation (6) determine the change in velocity vector $V_{b}$ from the rotation of the body frame with respect to the inertial frame. The angular velocity of the body frame with respect to the inertial frame is expressed in $\omega_{b}$.

Derivatives $i_{b}, j_{b}$ and $k_{b}$ can be found using (8):

$\frac{d}{d t_{i}} i_{b}=\omega_{b} \times i_{b}, \quad \frac{d}{d t_{i}} j_{b}=\omega_{b} \times j_{b}, \quad \frac{d}{d t_{i}} k_{b}=\omega_{b} \times k_{b}$

Substituting equations (7) and (8) to the right-hand side of equation (6), the following form was obtained (9):

$\frac{d V_{i}}{d t_{i}}=\frac{d V_{b}}{d t_{b}}+V_{x_{b}}\left(\omega_{b} \times i_{b}\right)+V_{y_{b}}\left(\omega_{b} \times j_{b}\right)+V_{z_{b}}\left(\omega_{b} \times k_{b}\right)$

Taking the last three conditions of the right side of the equation (9) before the parenthesis, the following forms of equations (10) and (11) were obtained:

$$
\begin{array}{r}
\frac{d V_{i}}{d t_{i}}=\frac{d V_{b}}{d t_{b}}+\omega_{b} \times\left(V_{x_{b}} i_{b}\right)+\omega_{b} \times\left(V_{y_{b}} j_{b}\right)+\omega_{b} \times\left(V_{z_{b}} k_{b}\right) \\
\frac{d V_{i}}{d t_{i}}=\frac{d V_{b}}{d t_{b}}+\omega_{b} \times\left(V_{x_{b}} i_{b}+V_{y_{b}} j_{b}+V_{z_{b}} k_{b}\right)
\end{array}
$$


In the light of the above, the desired relationship was obtained (12):

$$
\frac{d V_{i}}{d t_{i}}=\frac{d V_{b}}{d t_{b}}+\omega_{b} \times V_{b}
$$

The resulting compound can be used in equation (4) to express the temporal derivative of the velocity vector with respect to the body frame, resulting in (13):

$$
\Sigma F_{b}=m \frac{d V_{b}}{d t}+\omega_{b} \times\left(m V_{b}\right)
$$

By maintaining the equation of motion in fixed coordinate systems, to facilitate the derivation the force can be separated from the surface and the body as (14):

$$
F_{S}+W=m \frac{d V_{b}}{d t}+\omega_{b} \times\left(m V_{b}\right)
$$

The equations of translational motion are expressed in terms of their vector elements, starting from the terms related to the angular velocity, located on the right side of the equation (14) as (15):

$\omega_{b} \times\left(m V_{b}\right)=m\left|\begin{array}{lll}i_{x_{b}} & i_{y_{b}} & i_{z_{b}} \\ \omega_{x_{b}} & \omega_{y_{b}} & \omega_{z_{b}} \\ V_{x_{b}} & V_{y_{b}} & V_{z_{b}}\end{array}\right|=m\left[\begin{array}{l}\omega_{y b} V_{z b}-\omega_{z b} V_{y b} \\ \omega_{z b} V_{x b}-\omega_{x b} V_{z b} \\ \omega_{x b} V_{y b}-\omega_{y b} V_{x b}\end{array}\right]$

By substituting equation (15) for equation (14) and expanding the rest of words of vector elements, the following form of equation was obtained (16):

$$
\left\{\begin{array}{l}
F_{S_{x b}} \\
F_{S_{y b}} \\
F_{S_{z b}}
\end{array}\right\}+\left\{\begin{array}{l}
W_{x b} \\
W_{y b} \\
W_{z b}
\end{array}\right\}=m \frac{d}{d t}\left\{\begin{array}{l}
V_{x b} \\
V_{y b} \\
V_{z b}
\end{array}\right\}+m\left[\begin{array}{l}
\omega_{y b} V_{z b}-\omega_{z b} V_{y b} \\
\omega_{z b} V_{x b}-\omega_{x b} V_{z b} \\
\omega_{x b} V_{y b}-\omega_{y b} V_{x b}
\end{array}\right]
$$

The above equations of motion are expressed in terms of their vector elements in a way that allows dividing equations into longitudinal and lateral dynamics [22], [23], [24].

Equation (16) can be transformed as (17):

$$
m \frac{d}{d t}\left\{\begin{array}{l}
V_{x b} \\
V_{y b} \\
V_{z b}
\end{array}\right\}=\left[\begin{array}{l}
F_{S_{x b}}+W_{x b}-m \omega_{y b} V_{z b}+m \omega_{z b} V_{y b} \\
F_{s_{y b}}+W_{x b}-m \omega_{z b} V_{x b}+m \omega_{x b} V_{z b} \\
F_{S_{z b}}+W_{z b}-m \omega_{x b} V_{y b}+m \omega_{y b} V_{x b}
\end{array}\right]
$$

It should be noted that the derivation of equations is valid for drones with a constant mass. However, some UAV objects change in mass over time as a result of fuel combustion. Therefore, the equations of motion must take into account this stream of momentum.

Since the examined drone does not change mass over time, the time of changing the speed can be expressed as (18):

$$
\left\{\begin{array}{l}
\dot{V}_{x b} \\
\dot{V}_{y b} \\
\dot{V}_{z b}
\end{array}\right\}=\left[\begin{array}{l}
F_{S_{x b}}+W_{x b}-m \omega_{y b} V_{z b}+m \omega_{z b} V_{y b} \\
F_{S_{y b}}+W_{x b}-m \omega_{z b} V_{x b}+m \omega_{x b} V_{z b} \\
F_{S_{z b}}+W_{z b}-m \omega_{x b} V_{y b}+m \omega_{y b} V_{x b}
\end{array}\right]
$$

where: $F_{b}$ - is a pseudo-aerodynamic force.

The classic equation is replaced by the following equations (19), (20) and (21):

$$
V=\left\{\begin{array}{l}
V_{x b} \\
V_{y b} \\
V_{z b}
\end{array}\right\}=\left\{\begin{array}{c}
u \\
v \\
w
\end{array}\right\}=\left\{\begin{array}{c}
\text { axial velocity } \\
\text { sideslip velocity } \\
\text { normal velocity }
\end{array}\right\}
$$

$$
\begin{gathered}
\omega_{b}=\left\{\begin{array}{l}
\omega_{x b} \\
\omega_{y b} \\
\omega_{z b}
\end{array}\right\}=\left\{\begin{array}{l}
\mathcal{p} \\
q \\
r
\end{array}\right\}=\left\{\begin{array}{c}
\text { rolling rate } \\
\text { pitching rate } \\
\text { yawing rate }
\end{array}\right\} \\
m=W / g
\end{gathered}
$$

After substitution of flight dynamics entries in equation (18), the following form of equation was obtained (22):

$\left[\begin{array}{ccc}W / g & 0 & 0 \\ 0 & W / g & 0 \\ 0 & 0 & W / g\end{array}\right]=\left\{\begin{array}{l}\dot{u} \\ \dot{v} \\ \dot{v}\end{array}\right\}=\left\{\begin{array}{l}F_{x b}+W_{x b}+(r v-q w) W / g \\ F_{y b}+W_{y b}+(p w-r u) W / g \\ F_{z b}+W_{z b}+(q u-p v) W / g\end{array}\right\}$

\section{C.Identified nominal model and flight data}

This sub-section presents the nominal models identified using the least-squares recursive model BLS (Batch Least Squares) and BLS model with the error filtering model in "online" learning EFOL (Error Filtering Online Learning). These models are evaluated to match their flight data. The sine wave, double and single are used to evaluate the nominal models.

It should be remembered that the nominal models were built from training data with narrow maneuver data during the flight. The remaining maneuvers are used only to assess the characteristics of the nominal model. This is a standard procedure for assessing identical models and determines whether the nominal models identify all dynamic modes.

The nominal model BLS in equation (23) and BLS with the EFOL model (24) are presented in the context of linear longitudinal equations of motion.

$\left\{\begin{array}{c}\Delta \dot{u} \\ \Delta \dot{w} \\ \Delta \dot{q} \\ \Delta \dot{\theta}\end{array}\right\}=\left[\begin{array}{cccc}-0,18 & 0,19 & 0,18 & -9,8 \\ -0,76 & -5,13 & 14,05 & 0 \\ -0,11 & -1,89 & -1,13 & 0 \\ 0 & 0 & 1 & 0\end{array}\right]\left\{\begin{array}{c}\Delta u \\ \Delta w \\ \Delta q \\ \Delta \theta\end{array}\right\}+\left[\begin{array}{c}-0,43 \\ -18,12 \\ -37,53 \\ 0\end{array}\right] \Delta \delta e$
$\left\{\begin{array}{c}\Delta \dot{u} \\ \Delta \dot{w} \\ \Delta \dot{q} \\ \Delta \dot{\theta}\end{array}\right\}=\left[\begin{array}{cccc}-0,18 & 0,19 & 0,18 & -9,8 \\ -0,75 & -5,12 & 13,98 & 0 \\ -0,11 & -1,91 & -1,08 & 0 \\ 0 & 0 & 1 & 0\end{array}\right]\left\{\begin{array}{c}\Delta u \\ \Delta w \\ \Delta q \\ \Delta \theta\end{array}\right\}+\left[\begin{array}{c}-0,45 \\ -18,35 \\ -37,69 \\ 0\end{array}\right] \Delta \delta e$

When assessing the nominal models and comparing them to validated flight data, the nominal models are initiated with the flight data in time equal to 0 . The models are then excited using the elevator rudder flight data.

The paper presents three models of responses to input data of the flight data, where the responses of nominal models display characteristic results of the system identifier. The next figures (Figures 5-7) show three types of input signals.

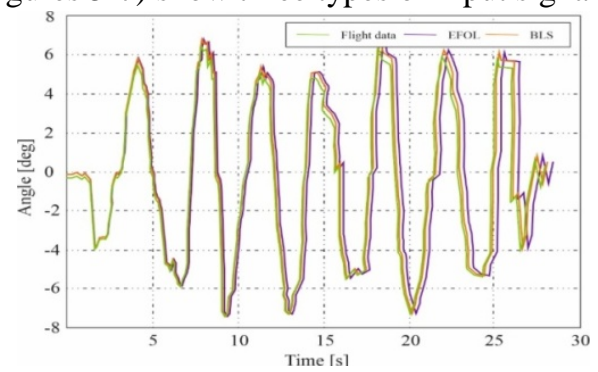

Fig. 5. Distribution of the sine wave 


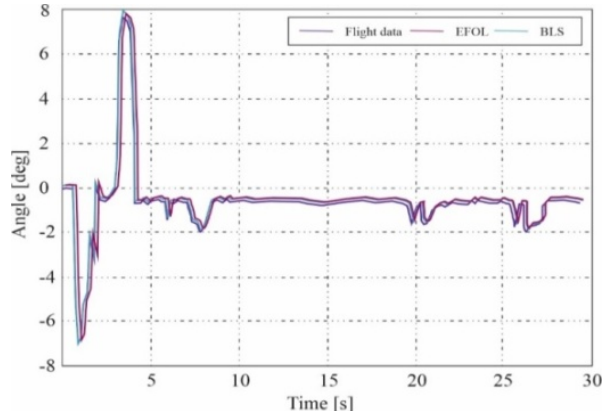

Fig. 6. Distribution of the doublet

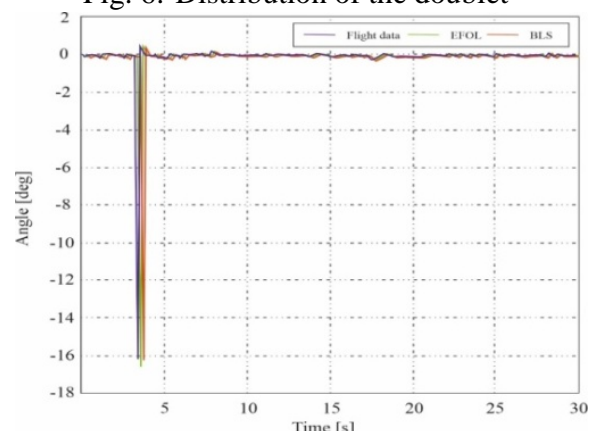

Fig. 7. Distribution of the singlet

\section{CONCLUSIONS}

System identification has been and will continue to be an invaluable tool for determining mathematical models that accurately simulate dynamic systems. This is particularly useful for manned and unmanned aerial vehicles where analytical solutions may not be sufficient, wind tunnel tests are not available, and CFD (Computational Fluid Dynamics) methods can be too time-consuming. There are many advantages of the UAV object identification system, but there are also several difficulties that need to be resolved appropriately [25], [26], [27].

The conclusions drawn are a direct result of struggling with kinematically consistent flight data and determining linear equations of long-term motion parameters. It is important that the data about flights were kinematically consistent, otherwise the system identifier will be ineffective.

The BLS and BLS with EFOL models positively identify linear longitudinal equations of motion parameters. The average value of these parameters is used to create nominal models. Nominal models contain flight data for sine maneuvers, but have a smaller set of double and single-point maneuvers.

A poor match corresponds to incorrect identifying the long period mode. A better match can be obtained using a double or single signal as part of the shaping of the data model.

Nominal models also do not predict vibrations of 1-2 [Hz], occurring in doublets and singlets. The causes of these oscillations should be investigated to see if they are the result of aerodynamics or the structure of the aircraft.

\section{REFERENCES}

[1] R. W. Beard, and T. W. McLain,Small Unmanned Aircraft: Theory and Practice," Princeton University Press, Princeton, NJ, 2012.
[2] L. Setlak, R. Kowalik, “Airplanes Compliant with the Concept of Electrified Aircraft,” Applied Sciences (Switzerland), Tom 9, Wydanie 8, 2019/1, EISSN:2076-3417.

[3] B. Ramos, D.S. Loubach, A.M. da Cunha, "Developing a distributed realtime monitoring system to track UAVs," IEEE Aerosp. Electron. Syst. Mag., Vol. 25, pp. 18-25, 2010.

[4] J. Dongwon, and T. Panagiotis, "Modeling and Hardware-in-the-loop Simulation fora Small Unmanned Aerial Vehicle," Proceedings of the 2007 AIAA Conference, 2007.

[5] L. Setlak, R. Kowalik, "Evaluation of the VSC-HVDC system performance in accordance with the more electric aircraft concept," IEEE Xplore, 28 June 2018.

[6] M. Kratky, V. Minarik, "The non-destructive methods of fight against UAVs," In Proceedings of the 2017 International Conference on Military Technologies (ICMT), Brno, Czech Republic, 31 May-2 June, pp. 690-694, 2017.

[7] F. O. Eke, "Dynamics of Variable Mass Systems," Tech. Rep., UC Davis, 1998.

[8] A. R. Plastino, and J. C. Muzzio, "On the Use and Abuse of Newton's Second Lawfor Variable Mass Problems," Celestial Mechanics and Dynamical Astronomy, Vol. 53, No. 3, pp. 227-232, 1992.

[9] Y. Nong, Z. Qi, and D. Lin, "System Identification of a Small Unmanned AerialVehicle Based on Time and Frequency Domain Technologies," Proceedings of the 2011, 9th World Congress on Intelligent Control and Automation, pp. 711-718, 2011.

[10] Y. Luo, H. Chao, L. Di, and Y. Chen, "Lateral Directional Fractional Order (pi)Control of a Small Fixed-wing Unmanned Aerial Vehicles: Controller Designs and Flight Tests," IET Control Theory \& Applications, Vol. 5, No. 18, pp. 2156, 2011.

[11] E. Atkins, R. Miller, T. Plet, K. Shaw, W. Ribbens, and D. Washabugh, "Solus: An Autonomous Aircraft for Flight Control and Trajectory Planning Research,” Proceedings of the 1998 American Control Conference, 1998.

[12] L. Setlak, R. Kowalik, "The effectiveness of on-board aircraft power sources in line with the trend of a more electric aircraft," IEEE Xplore, 28 June 2018.

[13] B.H. Sheu, C.C. Chiu, W.T. Lu, C.C. Lien, T.K. Liu, W.P. Chen, ”Dual-axis Rotary Platform with UAV Image Recognition and Tracking," Microelectron. Reliab., Vol. 95, pp. 8-17, 2019.

[14] M. Lort, A. Aguasca, C. López-Martínez, T.M. Marín, "Initial Evaluation of SAR Capabilities in UAV Multicopter Platforms,” IEEE J. Sel. Top. Appl. Earth Obs. Remote Sens., Vol. 11, pp. 127-140, 2018.

[15] P. Sebastain, L. Andrei, "Considerations Regarding Detection and Combat System for UAV’s,” Vol. 18, pp. 49-55, 2017.

[16] A. Kallapur, S. Ali, and S. Anavatti, "Application of Extended Kalman Filter Towards UAV Identification,” Autonomous Robots and Agents, Vol. 207, pp. 199-207, 2007.

[17] V. Stary, V. Krivanek, A. Stefek, "Optical detection methods for laser guided unmanned devices,” J. Commun. Netw., Vol. 20, pp. 464-472, 2018.

[18] M. Liu, G. Egan, and Y. Ge, "Identification of Attitude Flight Dynamics for an Unconventional UAV," Proceedings of the 2006 IEEE/RSJ International Conferenceon Intelligent Robots and Systems, pp. 3243-3248, 2006.

[19] L. Setlak, R. Kowalik, "Examination of the Unmanned Aerial Vehicle," ITM Web of Conferences, Tom 24, EDP Sciences ,(20 January 2019), eISSN: 2271-2097.

[20] H. Chao, Y. Luo, L. Di, and Y. Q. Chen, "Roll-channel Fractional Order Con-troller Design for a Small Fixed-wing Unmanned Aerial Vehicle," Control EngineeringPractice, Vol. 18, No. 7, pp. 761-772, 2010.

[21] D. T. Greenwood, "Principles of Dynamics," Prentice Hall, Upper Saddle River, NJ, 2nd ed., 1988.

[22] J. Nino, F. Mitrache, P. Cosyn, and R. Dekeyser, "Model Identification of a MicroAir Vehicle,” Journal of Bionic Engineering, Vol. 4, No. 4, pp. 227236, 2007.

[23] G. Eason, B. Noble, and I. N. Sneddon, "On certain integrals of LipschitzHankel type involving products of Bessel functions,” Phil. Trans. Roy. Soc. London, vol. A247, pp. 529-551, April 1955. (references)

[24] Z. Xu, R. Wei, X. Zhao, S. Wang, "Coordinated Stando Target Tracking Guidance Method for UAVs,” IEEE 2018, Vol. 6, pp. 59853-59859, 2018.

[25] D. Jung, E. Levy, D. Zhou, R. Fink, J. Moshe, A. Earl, and P. Tsiotras, "Designand Development of a Low-cost Test-bed for Undergraduate Education in UAVs," Proceedings of the 44th IEEE Conference on Decision and Control, pp. 2739-2744, 2005.

[26] L. Setlak, R. Kowalik, "Analysis, Mathematical Model and Simulation Tests of the Unmanned Aerial Vehicle Control System”, ITM Web of Conferences, Tom 24, EDP Sciences ,(20 January 2019), eISSN: 2271-2097.

[27] H. Oraizi, H. Soleimani, "Optimum pattern synthesis of non-uniform spherical arrays using the Euler rotation," IET Microw. Antennas Propag., Vol. 9, pp. 898-904, 2015. 